\title{
Rule differences, practice, and verbal solutions using a reception procedure in complete learning
}

\author{
EDW ARD M. DOCHERTY, LINDA J. INGISON, and JUDITH A. RESNICK \\ University of Wisconsin, Madison, Wisconsin 59706
}

\begin{abstract}
Rule differences in a complete learning (CL) task, using a reception procedure with neutral labels, were investigated over practice. Subjects were given 20 problems, in four blocks of five problems each. Within each block, problems representing the affirmative (Af), conjunctive $(\mathrm{Cj})$, conditional $(\mathrm{Cd})$, inclusive disjunctive (Id), and biconditional (Bd) rules were presented. The order of rule difficulty was found to be Af $=\mathrm{Cj}<\mathrm{Cd}<\mathrm{Id}<\mathrm{Bd}$. Performance improved through the third block of five problems, but there was no significant order of rule difficulty by practice interaction. The results for rule differences are inconsistent with previous research using the selection procedure. No currently available model of CL can account for differences in rule order under both selection and reception procedures. Verbal solutions were found to focus on the more homogeneous response category for the $\mathrm{Af}, \mathrm{Cj}$, and $\mathrm{Cd}$ rules. For the more difficult Id and Bd rules, verbal solutions focused equally frequently on the more homogeneous and the more heterogeneous response categories.
\end{abstract}

Systematic differences in rule difficulty form the basis for models of complete learning (CL; Docherty, Ingison, \& Gallagher, 1976; Hunt, Marin, \& Stone, 1966; Neisser \& Weene, 1962), as they do for rule learning (RL; Bourne, 1974; Salatas \& Bourne, 1974) and attribute identification (AI; Laughlin, 1968; Laughlin \& Jordan, 1967). Using a selection procedure in CL, Docherty et al. (1976) found highly stable rule differences over practice and conditions of neutral and directional response labels for the five "primary" rules. In addition, performance improved with practice, though order of rule difficulty did not change. Type of response label did not affect performance at all. The order of rule difficulty for the five "primary" rules was found to be exactly as predicted by Neisser and Weene on the basis of logical and hierarchical complexity: affirmative (Af) < conjunctive $(\mathrm{Cj})=$ conditional $(\mathrm{Cd}) \doteq$ inclusive disjunctive (Id) $<$ biconditional (Bd). It should be noted that under neutral labels a particular partition of stimuli into the two response categories defines both the primary rule and its complement, though in this study the primary rule is used to label the partition.

These rule differences may be specific to the selection procedure used. Laughlin (1969) found that in $\mathrm{AI}$ the order of rule difficulty varied with the use of reception or selection procedures. While findings in $\mathrm{AI}$ and $\mathrm{RL}$ are not necessarily generalizable to $\mathrm{CL}$, there is some evidence that rule differences in $\mathrm{CL}$

Send requests for reprints to Edward $M$. Docherty, now at Graduate School of Applied and Professional Psychology, Psychology Building, Busch Campus, Rutgers University, New Brunswick, New Jersey 08903 . Special appreciation is given to John D. Hammerly for his assistance in setting up, programming, and maintaining the computer arrangement used in this study. This paper is sponsored by P. G. Kepros, who takes full editorial responsibility for its contents. change when a reception procedure is used. Giambra (1974) found that $\mathrm{Cj}$ problems were significantly easier than Id problems in a reception CL study, though this result is limited to unpracticed subjects and only two of the five primary rules. Thus, the purpose of the present study was to evaluate differences among the five primary rules, over practice, using a reception CL procedure.

The effect of type of label may also vary with the procedure used. Docherty et al. (1976) found no effects for type of label in CL using selection procedures. Giambra (1974) used a reception paradigm in CL and found no significant difference in type of label (neutral or directional) for the Id rule. However, the $\mathrm{Cj}$ rule was significantly easier when directional labels were used than when neutral labels were used. Since subjects in this study solved only one problem, this result may be simply an example of the well-known conjunctive bias (Bruner, Goodnow, \& Austin, 1956). More importantly, for the purposes of this study, type of label did not change the order of rule difficulty. Since the order of rule difficulty was of primary importance in the present study, only neutral labels were used.

The use of neutral labels permits an interesting investigation of the type of verbal solutions subjects provide for a CL task. In a RL task using the Cd and Bd rules, Peters and Denny (1971) found that subjects more often phrased their solutions in terms of both response categories when the response labels were neutral (actually " $A$ " and " $B$ ") than when they were highly directional ("yes" and "no"). Under the directional labels, subjects' solutions tended to focus on the positive response category. However, in a CL task using neutral labels Giambra (1974) observed that, for the $\mathrm{Cj}$ rule, most subjects "indicated by their explicated method of stimulus classification that they 
eventually solved the problem by focusing on the label which appeared with the lesser frequency" (p.1158). The label with the lesser frequency represents what Bourne and Guy (1968) call the more homogeneous response category. No indication was given of the solutions provided for the Id rule in the Giambra study. In the present study verbal solutions were also obtained, allowing an examination of their form on the five primary rules.

\section{METHOD}

\section{Subjects and Design}

The subjects were 1613 - to 14-year-old paid volunteers, 8 males and 8 females.

The experimental design was a 2 by 4 by 5 repeated measures incorporating two sexes, four blocks of problems, and five rules within each block. Both blocks and rules were repeated measures. Number of errors in the first 100 trials was used as the dependent variable, and $\alpha$ was set equal to .05 .

\section{Materials}

The stimuli were four-dimensional geometric patterns with three values on each dimension. The dimensions were color (blue, red, or yellow), form (square, hexagon, or triangle), size (small, medium, or large), and number of figures (one, two, or three). The 81 instances representing all combinations of the four ternary-valued dimensions were displayed on slides and projected on a screen before the subject.

\section{Task and Procedure}

Subjects were given four blocks of five problems each. Within each block, problems representing each of the $\mathrm{Af}, \mathrm{Cj}, \mathrm{Cd}$, Id, and $\mathrm{Bd}$ rules were presented in random order. Relevant attributes for each problem were determined such that all attributes were used approximately equally, and no two successive problems had the same pair of relevant attributes. The sequence of stimuli was determined such that no single attribute appeared three times in a row, and within every nine trials the number of instances in each truth table category was proportional to their frequency in the stimulus population. Response labels were assigned randomly to one of the two reponse categories.

A CL reception procedure was used. Each subject was seated in front of a console consisting of a rear projection screen and a computer terminal with a keyboard and cathode ray tube (CRT) screen. The keyboard was masked so that only the Return key and two response keys, labeled * and \#, were visible. The rule, relevant attributes, and stimulus sequence were determined by a computer program within the constraints listed above. A random-access slide projector, controlled by the computer program, was used to present the stimuli. The nature and characteristics of the stimuli, task, and use of the keyboard were completely described to the subjects. The subjects responded to each siide by pressing the desired response key. The correct response was then presented on the CRT screen. When the subjects were ready to go on to the next slide, they

Table 1

Mean Number of Errors for Five Rules on Four Repeated Blocks of Problems

\begin{tabular}{crrcccr}
\hline & \multicolumn{5}{c}{ Rule } & \\
\cline { 2 - 6 } 3lock & Af & \multicolumn{1}{c}{ Cj } & Cd & Id & Bd & Total \\
\hline 1 & 10.00 & 16.13 & 22.69 & 28.63 & 37.75 & 23.04 \\
2 & 6.75 & 9.06 & 18.31 & 29.06 & 35.38 & 19.71 \\
3 & 4.31 & 9.44 & 14.13 & 22.69 & 33.13 & 16.74 \\
4 & 5.75 & 8.25 & 14.50 & 19.31 & 27.19 & 15.00 \\
Total & 6.70 & 10.72 & 17.41 & 24.92 & 33.36 & \\
\hline
\end{tabular}

Table 2

Frequency of Verbal Solutions Based on Homogeneous or Heterogeneous Response Categories for Five Rules

\begin{tabular}{cccc} 
& \multicolumn{2}{c}{ Type of Response Category } & \\
\cline { 2 - 3 } Rule & Homogeneous & Heterogeneous & Total \\
\hline Af & 44 & 18 & 62 \\
Cj & 59 & 2 & 61 \\
Cd & 51 & 9 & 60 \\
Id & 34 & 28 & 62 \\
Bd & 27 & 32 & 59 \\
\hline
\end{tabular}

pressed the Return key. Subjects continued on each problem until they reached a criterion of 16 consecutive correct choices, or 100 trials. Each subject was then required to provide a complete verbal statement of the rule. If the subject went through 100 trials without getting 16 in a row correct, the solution was presented on the CRT screen, indicating the correct classification for every combination of attributes from the relevant dimensions. The subject then proceeded for another 50 trials or 16 consecutive correct classifications. The problem was then terminated and a new one begun. Subjects completed the 20 problems in two to three $11 / 2-h$ sessions over a period of not more than 6 days.

\section{RESULTS}

A split-plot analysis of variance was performed on number of errors in the first 100 trials. The main effect for Rule was significant, $F(4,56)=38.50$, $\mathrm{p}<.01$. The means for the five rules are presented in Table 1. The Newman-Keuls post hoc comparison technique, with $\alpha=.05$ for all ordered sets of means, was used to test the differences between means for the five rules. The obtained rule differences were Af $=\mathrm{Cj}<\mathrm{Cd}<$ Id $<\mathrm{Bd}$.

The main effect for Block was also significant, $F(3,42)=11.76, p<.01$. The means for each block are also presented in Table 1 . Neuman-Keuls post hoc comparisons, with $\alpha=.05$, indicated that Block $1>$ Block $2>$ Block $3=$ Block 4 .

The main effect for Sex was not significant, $F(1,14)<1$, and there were no significant interactions.

The types of verbal solutions provided by the subjects are presented in Table 2. Solutions using one or both response categories were not differentiated because of an ambiguity in the instructions which confounded this distinction. Type of response category was defined as homogeneous or heterogeneous according to Bourne and Guy (1968), based on the number of stimuli in each response category. In the verbal solutions, type of response category was simply the category mentioned exclusively when only one category was provided by the subject, or the category mentioned first when both response categories were included in the solution. Total number of solutions does not add up to 64 (four problems times 16 subjects for each rule) because some verbal solutions did not fit into either the homogeneous or heterogeneous categories. The frequencies in Table 2 are based on nonindependent 
observations (four repeated observations on each of 16 subjects) so no statistical tests were used to analyze the data. However, visual inspection of Table 2 makes it clear that, for the $\mathrm{Af}, \mathrm{Cj}$, and $\mathrm{Cd}$ rules, the homogeneous category was mentioned exclusively or first more frequently than the heterogeneous category. For the Id and Bd rules the two categories were mentioned exclusively or first with approximately equal frequency.

\section{DISCUSSION}

Using a reception procedure, the order of rule difficulty in $C L$ was found to be $\mathrm{Af}=\mathrm{Cj}<\mathrm{Cd}<\mathrm{Id}<\mathrm{Bd}$. A previous study (Docherty et al., 1975) found the rule order for a selection procedure to be $\mathrm{Af}<\mathrm{Cj}=\mathrm{Cd}=\mathrm{Id}<\mathrm{Bd}$. The latter results fit the Neisser and Weene (1962) predictions exactly, while the results for the present study clearly do not. These results are also inconsistent with the predictions of Hunt, Marin, and Stone (1966), though Docherty et al. (1976) showed that the Hunt et al. predictions are little different from those of Neisser and Weene. The results of the present study are consistent with those of Giambra (1974), who also used a reception procedure, in that both studies found the $\mathrm{Cj}$ rule easier than the Id rule. There is no obvious interpretation of the discrepant results for reception and selection, especially in view of the stability of these rule differences over practice and type of response label used. Nor is there any obvious reason why the Neisser and Weene (1962) predictions should hold under the selection but not the reception procedures. In fact. Neisser and Weene provide more of a schematic structure than a real model of the $C L$ process. It seems, however, that different strategies are probably being used when the subject has stimuli presented to him than when he is free to choose the sequence of stimuli, and that different models will be required to describe the two situations.

There was a main effect for practice, in the present study, with performance improving over the first three blocks of problems, but there was no Rule Order by Practice interaction. This latter finding is consistent with the Docherty et al. (1976) study and supports their conclusion that Neisser and Weene (1962) found a Rule Order by Practice interaction because of their exceptionally difficult stimulus materials. However, under the selection procedure used by Docherty et al. (1976), there was no improvement in performance after the second block of problems, while in the reception procedure used here, performance improved through the third block of trials. Though performance leveled out by the fourth block of problems, it is possible that further practice would lead to further improvement in CL.

The data on verbal solutions suggests that for neutral labels and relatively easy rules, the subject focuses on the more homogeneous of the two response categories. For the two most difficult rules, however, both the more homogeneous and the more heterogeneous response categories are focused upon with approximately equal frequency. These results are consistent with Seggie's (1969) suggestion that, for the $\mathrm{Cj}$ and, apparently, other relatively easy rules, the subjects need learn only part of the concept (in this case the more homogeneous category), while for more difficult rules such as the $\mathrm{Dj}$. subjects are more likely to use all the information presented. Seggie's (1969) results, however, are not comparable to those presented here because of procedural differences and other difficulties pointed out by Giambra (1974). Comparison of these results for verbal solutions with those for a selection procedure are not possible at present, for verbal solutions were not analyzed in the Docherty et al. (1976) study.

\section{REFERENCES}

Bourne, L. E., JR. An inferential model for conceptual rule learning. In R. L. Solso (Ed.), Theories in cognitive psychology: The Loyola symposium. New York: Wiley, 1974.

Bourne, L. E., JR., \& GUY, D. E. Learning conceptual rules: II. The role of positive and negative instances. Journal of Experimental Psychology, 1968, 77, 488-494.

Bruner, J. S., Goodnow. J. J., \& Austin. G. A. A study of thinking. New York: Wiley. 1956.

Docherty, E. M., Ingison, L. J., \& Gallagher, D. Rule differences, practice, and response label in complete learning. Bulletin of the Psychonomic Society, 1976, in press.

Giambra, L. M. Labels for response categories: Do neutral labels make conjunctive and inclusive disjunctive concepts equally difficult? Psychological Reports, 1974, 35, 1155-1159.

Hunt, E. B., Marin, J., \& Stone, P. J. Experiments in induction. New York: Academic Press, 1966.

Laughlin, P. R. Focusing strategy for eight concept rules. Journal of Experimental Psychology, 1968, 77, 661-669.

Laughlin, P. R. Selection versus reception paradigms as a function of memory, concept rule, and concept universe. Journal of Educational Psychology, 1969, 60, 267-273.

Laughlin. P. R., \& Jordan, R. M. Selection strategies in conjunctive, disjunctive, and biconditional concept attainment. Journal of Experimental Psychology, 1967. 75. 188-193.

Neisser, U., \& WeENe, P. Hierarchies in concept attainment. Journal of Experimental Psychology, 1962, 64, 640-645.

Peters, K. G., \& Denny, J. P. Labeling and memory effects on categorizing and hypothesizing behavior for biconditional and conditional conceptual rules. Journal of Experimental Psychology, 1971, 87, 229-233.

Salatas, H., \& Bourne, L. E., JR. Learning conceptual rules: III. Processes contributing to rule difficulty. Memory \& Cognition, 1974, 2, 549-553.

SEgGIE. J. L. Levels of learning involved in conjunctive and disjunctive concepts. Australian Journal of Psychology. 1969. 21. 325-333.

(Received for publication May 13, 1976.) 